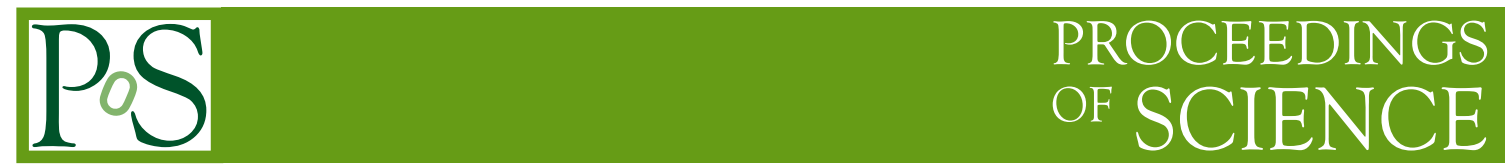

\title{
Searches for dark matter with CMS
}

\section{Andreas Albert* for the CMS collaboration}

III. Physikalisches Institut A, RWTH Aachen University, Aachen, Germany

E-mail: albertephysik.rwth-aachen.de

Searches in CMS for dark matter in final states with invisible particles recoiling against visible states are presented. Various topologies and kinematic variables are explored, including jet substructure as a means of tagging heavy bosons. The focus of the talk is the recent results obtained using data collected at Run-II of the LHC.

The 39th International Conference on High Energy Physics (ICHEP2018)

4-11 July, 2018

Seoul, Korea

${ }^{*}$ Speaker. 
The origin of Dark Matter (DM) in the universe is one of the most pressing questions in physics today. Astrophysical evidence seems consistent with DM being composed of particles. The CMS collaboration [1] pursues an active search program for the production of such particles at the CERN LHC, and formulates search results in the context of simplified models, which contain the minimal beyond-the-standard-model (BSM) particle content necessary to describe the signatures of interest. The minimal models with only one fermion DM particle and one BSM boson which supplies the interaction between the standard model (SM) and dark sectors (termed the "mediator") are described by four parameters: the mediator mass $m_{\text {med }}$ and the dark matter mass $m_{\mathrm{DM}}$, as well as the couplings of the mediator to quarks $g_{\mathrm{q}}$ and DM $g_{\mathrm{DM}}[2]$.

Since the CMS detector cannot detect DM particles directly, searches rely on missing transverse momentum $p_{T}^{\text {miss }}$ in association with a specific reconstructed "tag" signature". All results discussed here use a dataset corresponding to $35.9 \mathrm{fb}^{-1}$ of proton-proton collisions at $\sqrt{s}=13 \mathrm{TeV}$ recorded with the CMS detector in 2016. Additionally, a result for a leptoquark (LQ) mediator is presented which additionally makes use of a dataset corresponding to $41.5 \mathrm{fb}^{-1}$ collected in 2017.

The "monophoton" search [3] relies on events with a photon with $p_{T}>175 \mathrm{GeV}$ and $p_{T}^{\text {miss }}>$ $170 \mathrm{GeV}$ and determines the presence of a signal using an analysis of the shape of the $p_{T}(\gamma)$ distribution. As a major improvement over previous analyses, the shapes and normalizations of the dominant irreducible background (BG) contributions from $\mathrm{V} \gamma$ processes (where $\mathrm{V}=\mathrm{W}$ or $\mathrm{Z}$ ) are estimated from data. Control regions (CRs) target $\mathrm{V} \gamma$ production in events with reconstructed charged leptons as a proxy for the lepton-less signal region (SR). Using transfer factors from simulation with dedicated theoretical uncertainty estimates, a maximum-likelihood (ML) fit allows to robustly constrain the BG contributions. This novel technique provides a significant enhancement in analysis sensitivity. Specialized photon identification and isolation requirements are employed to suppress BGs from non-prompt photons, with residual contributions being estimated from data. The data in the SR are found to agree with the BG-only hypothesis and new spin-1 mediators can be excluded up to $m_{\text {med }} \approx 950 \mathrm{GeV}$ for $g_{\mathrm{q}}=0.25$ and $g_{\mathrm{DM}}=1.0$ (cf. Fig. 1).

A search for DM production in association with a $\mathfrak{t} \bar{t}$ system [4] relies on high-multiplicity final states. To target all possible top quark decay chains, events with large $p_{T}^{\text {miss }}$ are categorized based on the number of charged leptons, jets and $b$ jets. Further categorization into multiple SRs and CRs is performed based on the kinematic properties of the reconstructed top quark system. In all cases, the leading $\mathrm{BG}$ is $\mathrm{SM} t \bar{t}$ production. Using $p_{T}^{\text {miss }}$ as the main discriminating variable, a combined ML fit is employed to constrain the BG components and extract a possible signal in the same manner as in the monophoton analysis. No significant deviation from the BG prediction is observed. The $t \bar{t}$ search is especially sensitive to mediators with enhanced couplings to heavy fermions. Assuming $g_{\mathrm{q}}=g_{\mathrm{DM}}=1$, mediator masses of up to $165 \mathrm{GeV}(220 \mathrm{GeV})$ can be excluded in the scalar (pseudoscalar) case, which are the strongest bounds to date.

In order to probe the parameter space with $m_{\mathrm{DM}}>m_{\text {med }} / 2$, searches for mediator decays to $\mathrm{SM}$ particles, such as bottom quarks, are used. Mediator decays to $\mathrm{b} \overline{\mathrm{b}}$ pairs are challenging to detect over large SM jets BGs [5]. BG rejection is achieved by focusing on high- $p_{T}$ mediator candidates, the decay products of which are then collimated and are reconstructed as a single large-radius

\footnotetext{
${ }^{1}$ Missing transverse momentum is calculated as the magnitude of the vector sum of all reconstructed particle momenta.
} 

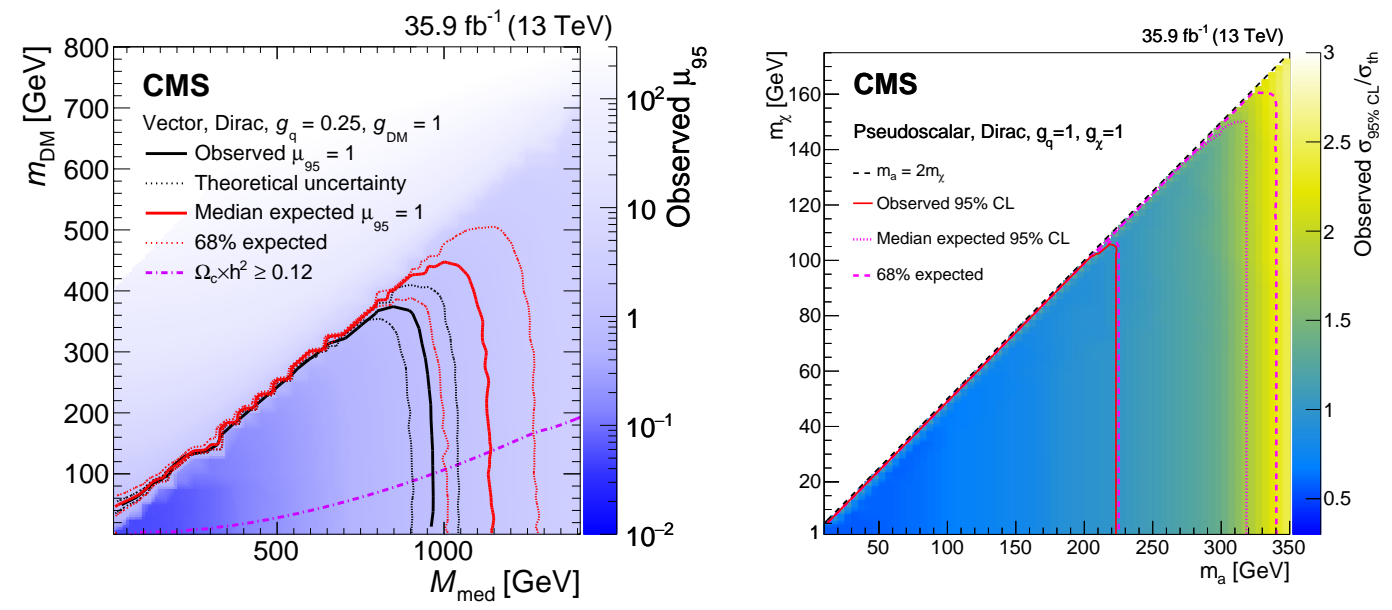

Figure 1: Exclusion limits for DM with a vector mediator from the monophoton analysis [3] (left) and the pseudoscalar mediator in the DM+t⿱亠t analysis [4] (right).

jet. A multivariate algorithm ("double b tagger") is applied to determine whether candidate jets have properties consistent with those of a jet consisting of two bottom quarks. A possible signal is extracted in categories of $p_{T}(j)$ using the distribution of the jet mass $m_{\mathrm{SD}}$. The main BG from QCD multijet production is estimated in a fully data-driven way from a control sample of events failing the double $\mathrm{b}$ tag requirement, with a transfer function parameterized as a polynomial in $p_{T}(j)$ and $\left.\rho=\log \left(m_{\mathrm{SD}}^{2} / p_{T}(j)^{2}\right)\right)$, which is obtained from a fit to data. Minor BG components from $\mathrm{V}, \mathrm{H}$ and $\mathrm{t} \overline{\mathrm{t}}$ production are estimated from simulation with data-driven normalization corrections. Depending on the mediator mass hypothesis, quark coupling modifiers as low as $g_{\mathrm{q}}=2\left(g_{\mathrm{q}}=3\right)$ can be excluded in the pseudoscalar (scalar) coupling case, providing the first constraints on this topology for a mediator with $m_{\text {med }}<325 \mathrm{GeV}$.

The mono-Higgs topology is studied in the $\mathrm{H}(\mathrm{b} \overline{\mathrm{b}})$ channel [6], which profits from a large branching fraction. Following a similar strategy as the direct mediator search, the Higgs boson is reconstructed from double $\mathrm{b}$ tagged large-radius jets with $p_{T}(j)>200 \mathrm{GeV}$ and $100<m_{\mathrm{SD}}<$ $150 \mathrm{GeV}$. The leading BG processes are $V$ and $t \bar{t}$ production, which are estimated in a scheme very similar to the monophoton analysis: While events in the SR are required to have no charged leptons, events with at least one charged lepton are sorted into CRs based on the number of charged leptons, their flavors and the presence of a $b$ tagged jet to separate the main BG components. BG and signal components are then once again determined in a simultaneous ML fit to the SR and CRs. Since no significant signal contribution is observed, exclusion limits are set - among others - in the baryonic Z' model, which contains direct couplings of the mediator and SM Higgs particle [2]. For low values of $m_{\mathrm{DM}}$, the baryonic $\mathrm{Z}$ ' hypothesis is excluded up to a mass of $1.6 \mathrm{TeV}$, which is the most stringent bound on this scenario to date.

DM production from LQ decays is probed in an analysis of events with a muon, a jet and large $p_{T}^{\text {miss }}$ [7]. Suppression of the leading BG contributions from the $t \bar{t}$ and $\mathrm{W}$ processes is achieved by requiring that no $\mathrm{b}$ tagged jets be present and the transverse mass of the muon- $p_{T}^{\text {miss }}$ system be larger than $500 \mathrm{GeV}$. The remaining BG contributions are estimated from simulation, with datadriven normalization corrections determined from dedicated CRs. Signal extraction is performed 

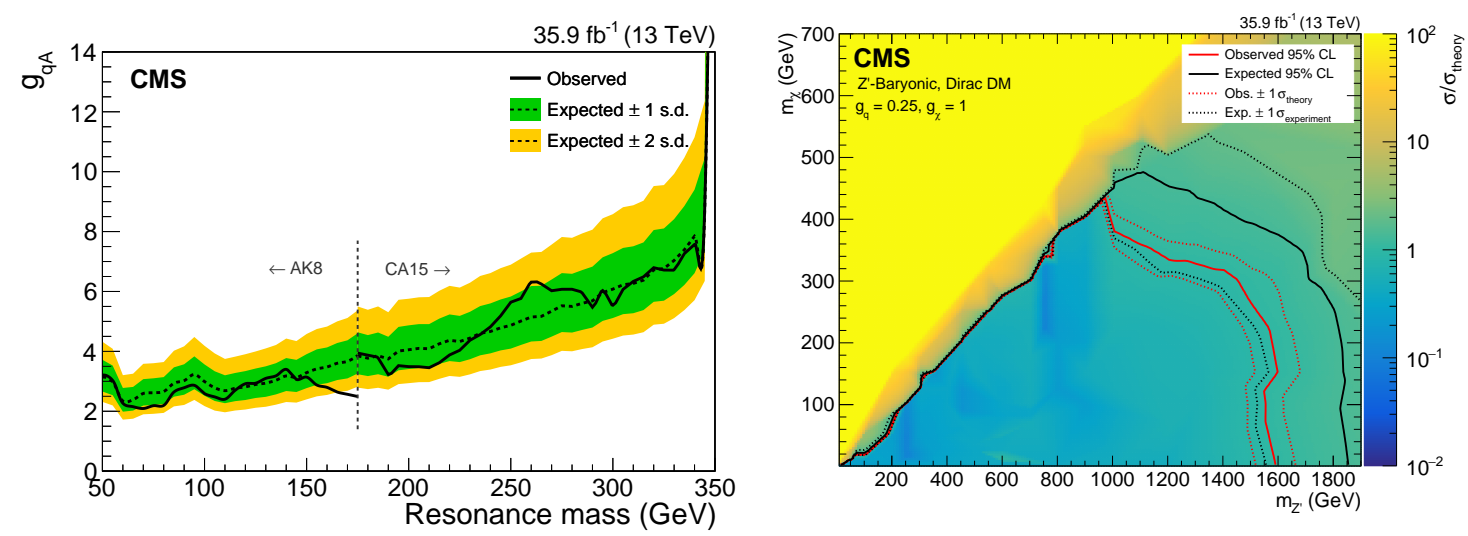

Figure 2: Exclusion limits for a pseudoscalar mediator from the mediator search in $b \bar{b}$ events [5] (left) and the baryonic Z' model from the Mono-Higgs search [6] (right).

differentially in the muon-jet invariant mass, and the signal contribution is found to be consistent with zero. The results are interpreted in a model with a LQ-like mediator, a new unstable fermion $\mathrm{X}$, and a fermionic DM candidate. Assuming a small mass splitting $m_{\mathrm{X}} / m_{\mathrm{DM}}-1=10 \%$, mediator masses between 800 and $1200 \mathrm{GeV}$ are excluded.

In summary, the CMS collaboration has presented a number of new search results for collider production of DM. A continuously growing recorded dataset and innovative experimental techniques allow the search sensitivity to be enhanced significantly. In the absence of a discovery, stringent bounds are set on a variety of signal hypotheses.

\section{References}

[1] CMS Collaboration, S. Chatrchyan et al., The CMS experiment at the CERN LHC, JINST 3 (2008) S08004.

[2] D. Abercrombie et al., Dark matter benchmark models for early LHC run-2 searches: Report of the ATLAS/CMS dark matter forum, 1507.00966.

[3] CMS Collaboration, A. M. Sirunyan et al., Search for new physics in final states with a single photon and missing transverse momentum in proton-proton collisions at $\sqrt{s}=13 \mathrm{TeV}$, Submitted to: JHEP (2018) [1810.00196].

[4] CMS Collaboration, A. M. Sirunyan et al., Search for dark matter particles produced in association with a top quark pair at $\sqrt{s}=13$ TeV, Submitted to: Phys. Rev. Lett. (2018) [1807. 06522].

[5] CMS Collaboration, A. M. Sirunyan et al., Search for low-mass resonances decaying into bottom quark-antiquark pairs in proton-proton collisions at $\sqrt{s}=13 \mathrm{TeV}$, Submitted to: Phys. Rev. (2018) [1810.11822].

[6] CMS Collaboration, Search for associated production of dark matter with a Higgs boson that decays to a pair of bottom quarks, Physics Analysis Summary CMS-PAS-EXO-16-050, CERN, Geneva, 2018. http://cds.cern.ch/record/2628473.

[7] CMS Collaboration, Search for dark matter in final states with a leptoquark and missing transverse momentum in proton-proton collisions at 13 TeV, Physics Analysis Summary CMS-PAS-EXO-17-015, CERN, Geneva, 2018. http://cds.cern.ch/record/2628478. 